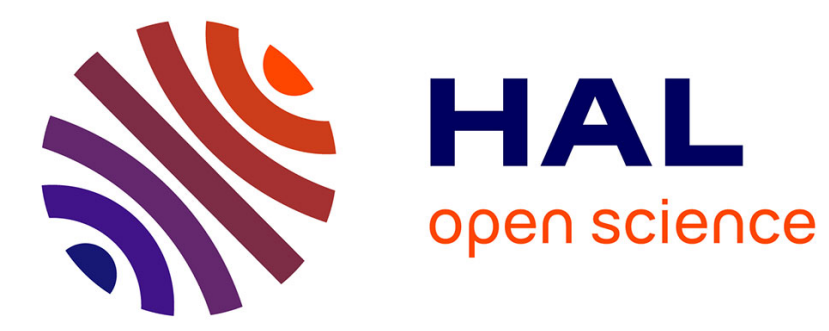

\title{
Les apports de la géochronologie à la connaissance du climat
}

\author{
Martine Paterne
}

\section{To cite this version:}

Martine Paterne. Les apports de la géochronologie à la connaissance du climat. Clefs CEA, 2009, (Terre et Environnement), 57, pp.12-16. hal-03325999

\section{HAL Id: hal-03325999 \\ https://hal.science/hal-03325999}

Submitted on 25 Aug 2021

HAL is a multi-disciplinary open access archive for the deposit and dissemination of scientific research documents, whether they are published or not. The documents may come from teaching and research institutions in France or abroad, or from public or private research centers.
L'archive ouverte pluridisciplinaire HAL, est destinée au dépôt et à la diffusion de documents scientifiques de niveau recherche, publiés ou non, émanant des établissements d'enseignement et de recherche français ou étrangers, des laboratoires publics ou privés. 


\section{Les apports de la géochronologie à la connaissance du climat}

Pour reconstruire l'évolution du climat d'une période donnée et replacer les événements dans un cadre chronologique global, l'exploitation des archives climatiques impose de jongler entre des méthodes de datation très variées. La mesure de la radioactivité par différentes techniques y joue souvent un rôle central.

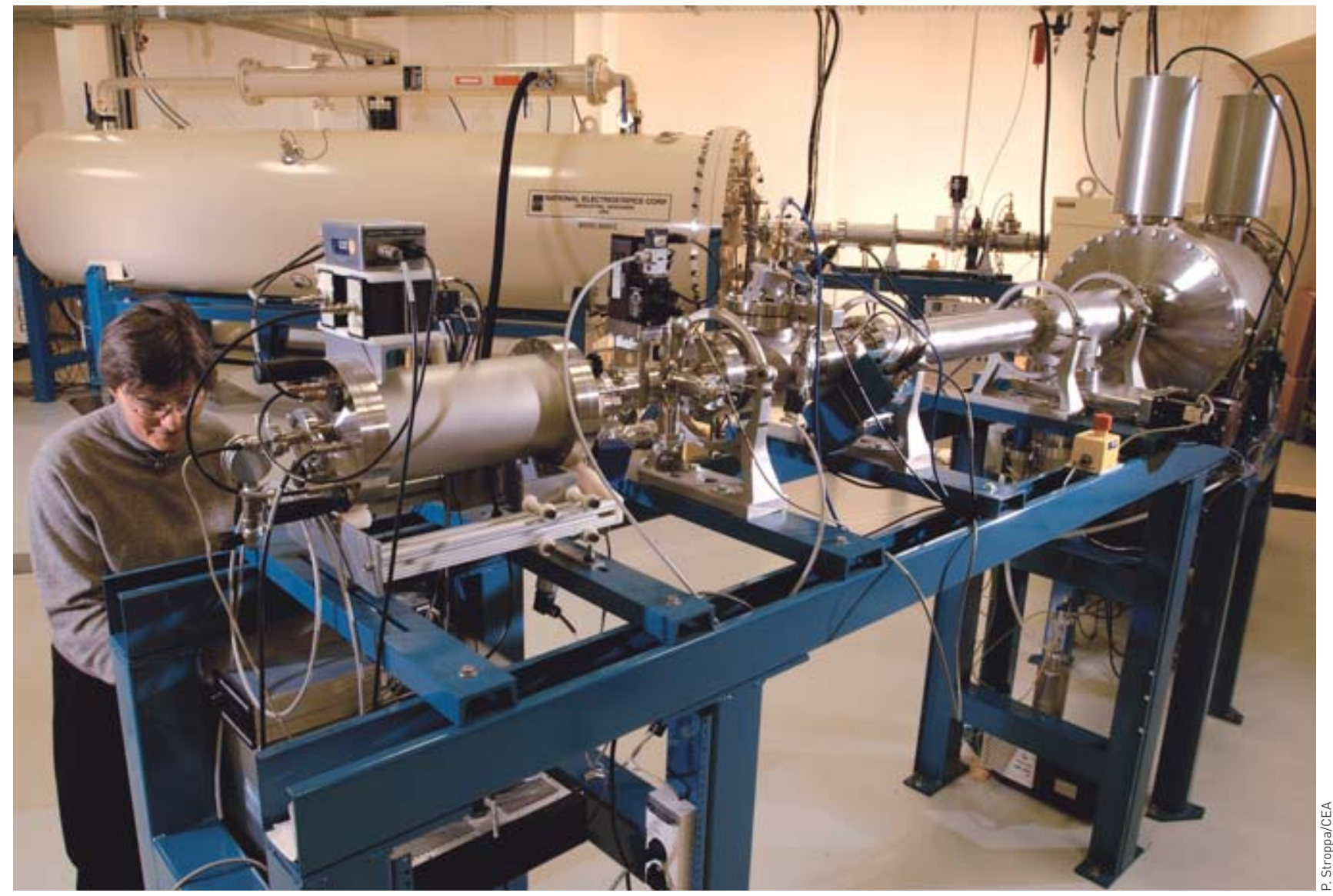

Vue générale du spectromètre de masse par accélérateur Artémis, installé sur le Centre de Saclay, qui permet la datation par le carbone 14 d'échantillons jusqu'à environ 50000 ans. Grâce aux diverses techniques de datation, toutes les informations issues des archives climatiques vont

s'assembler, telles les pièces d'un puzzle,

et se conforter pour

reconstruire l'évolution climatique d'une période donnée. e climat de la Terre varie naturellement avec des —constantes de temps allant de plusieurs centaines de millions d'années à la saison. Dès le XIx ${ }^{e}$ siècle, les géologues avaient apporté la preuve de l'existence de périodes glaciaires et interglaciaires dans le passé, en identifiant de vastes dépôts morainiques (1) au nord des continents américain et européen. Il restait cependant à connaître l'âge de ces changements climatiques qui caractérisent le Pléistocène, époque du Quaternaire couvrant environ 1,8 million d'années. Ceci devint possible avec la découverte et la mise au point de méthodes de mesures précises de la radioactivité, et le développement des techniques de carottages sédimentaires toujours plus profonds, que ce soit sur le continent, dans l'océan ou dans les glaces pour "remonter" le temps.

La géochronologie a pour objectif essentiel de replacer l'évolution de la Terre, et donc du climat, dans un cadre spatio-temporel unitaire. Elle est aussi une aide précieuse à la prévision de l'évolution du climat, car elle rend possible de confronter les différentes théories émises sur les causes et les mécanismes de la variation des climats passés. La réalisation de ce cadre chronologique global n'est pas simple, car elle repose sur un large éventail de méthodes de datation et sur leur croisement, ce qui permet de tester la validité des âges obtenus pour un même événement. Une difficulté supplémentaire réside également dans la diversité des matériaux datables, qui vont des cristaux inorganiques, quartz, feldspath, calcite ou aragonite (2), aux restes biogéniques, tels les coquillages, les tests (ou squelettes) de foraminifères ou bien les végétaux.

(1) Moraine: amas de débris minéraux transportés par un glacier ou par une nappe de glace et qui, au moment de la fonte, sont déposés, généralement à la même altitude, formant un empilement rocheux.

(2) Le carbonate de calcium $\left(\mathrm{CaCO}_{3}\right)$, constituant majeur du calcaire, de la craie, du marbre mais également des coquilles d'animaux marins et des escargots, cristallise naturellement sous deux formes principales: l'aragonite et la calcite. 


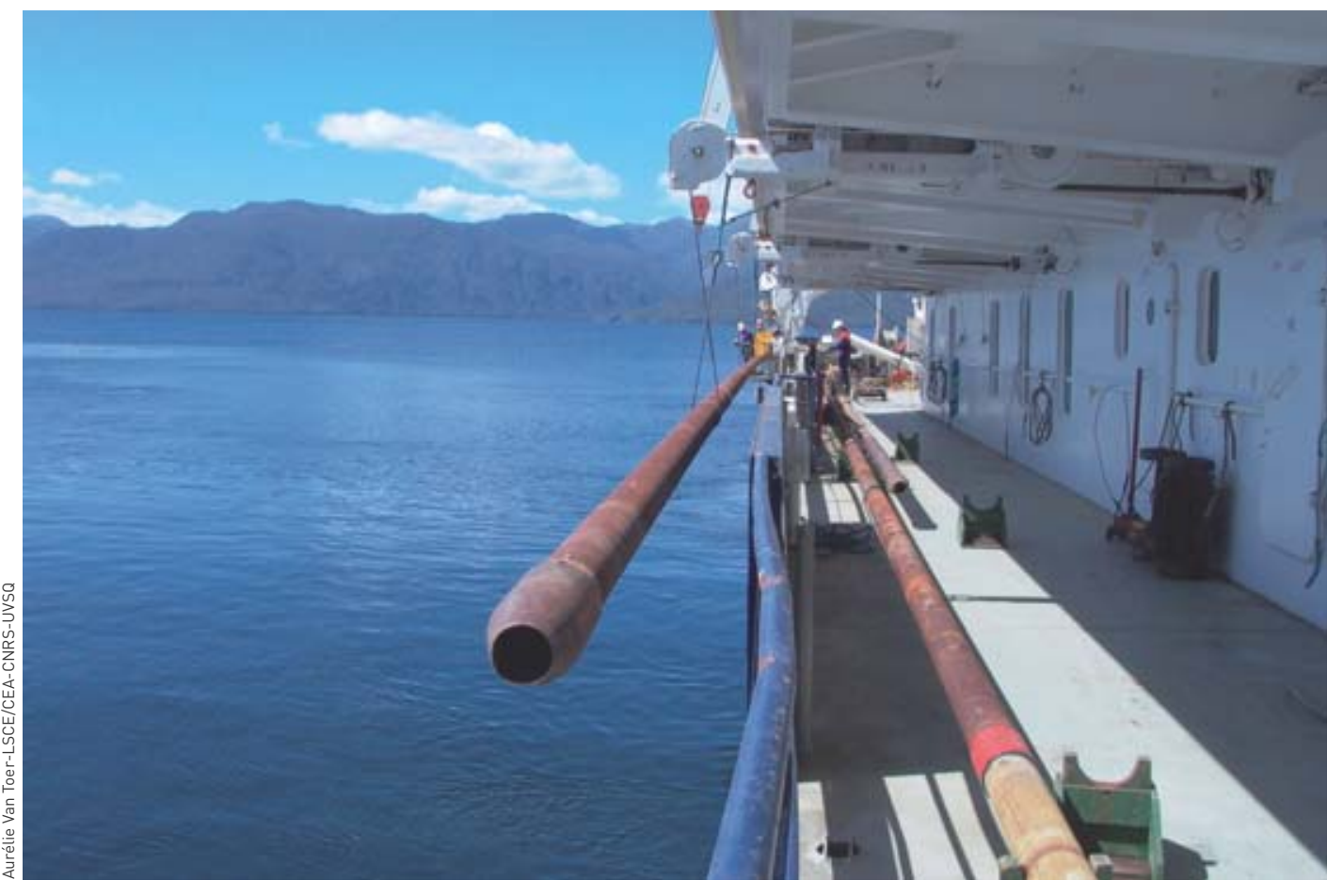

\section{Les techniques de datation appropriées} au Pléistocène

Les méthodes de datation appliquées au paléoclimat du Pléistocène sont généralement subdivisées en deux groupes: les méthodes de datations absolues, incluant les mesures de la radioactivité sensu lato, et les méthodes relatives. Ces dernières sont basées sur la corrélation de signaux physico-chimiques ou biologiques qui sont caractéristiques à l'échelle globale ou régionale dans les archives sédimentaires. Dans les faits, elles bénéficient des avancées de chacune d'entre elles. Les méthodes s'appuyant sur la radioactivité s'adressent à la datation d'événements ponctuels mis en place dans des temps géologiquement courts, identifiables ou non à l'échelle du globe. Ces datations servent de cadre chronologique à l'élaboration des échelles de temps relatives.

\section{Compter avec la dendrochronologie}

Les âges absolus ou calendar ages sont obtenus à partir du comptage direct de formations annuelles. Ce comptage est appliqué aux cernes de croissance annuelle des arbres par la méthode de dendrochronologie, aux dépôts annuels, bi-saisonniers, ou varves (3) dans les sédiments lacustres et marins, ou bien encore à ceux des couches de neige.

\section{Mesurer la radioactivité naturelle}

Avec le temps, les atomes radioactifs se désintègrent en formant des nucléides stables ou eux-mêmes radioactifs, lesquels se désintégreront à leur tour pour donner d'autres nucléides, et ainsi de suite. Les périodes de désintégration different selon les nucléides, ce qui permet d'appréhender la variabilité climatique sur plusieurs gammes de temps. Les nucléides radio-

(3) Varve (du suédois varvig signifiant rayé) : sédiment lacustre fait de dépôts alternativement fins et grossiers, déposé en avant des glaciers.

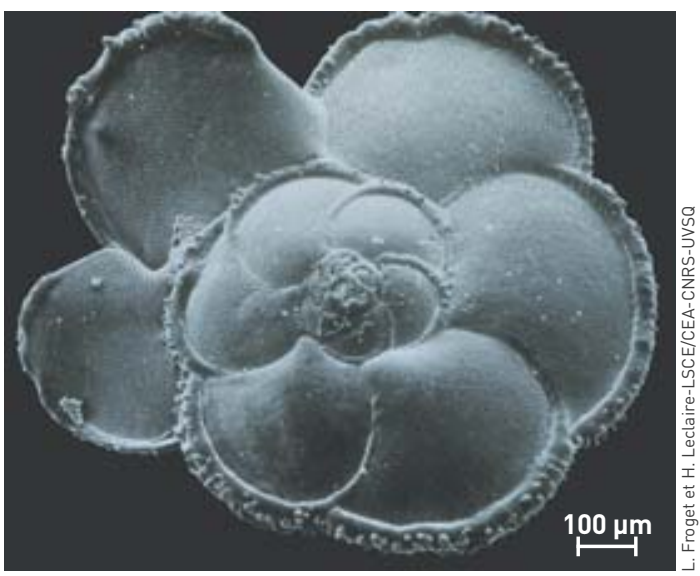

Globorotalia menardii fimbriata, foraminifère planctonique évoluant dans les eaux superficielles des océans. Des foraminifères vivent et meurent dans les eaux océaniques. Leurs coquilles sont retrouvées dans les sédiments marins.

actifs naturels sont présents sur la Terre depuis sa formation, tels l'uranium $238\left({ }^{238} \mathrm{U}\right)$, l'uranium 235 $\left({ }^{235} \mathrm{U}\right)$ et le thorium $232\left({ }^{232} \mathrm{Th}\right)$, ou le potassium $40\left({ }^{40} \mathrm{~K}\right)$, ou bien ils sont produits continuellement par l'action du rayonnement cosmique sur des noyaux dans la haute atmosphère, comme le carbone $14\left({ }^{14} \mathrm{C}\right)$ et le béryllium $10\left({ }^{10} \mathrm{Be}\right)$, appelés pour cette raison isotopes cosmogéniques.

Les méthodes potassium-argon (K/Ar) et argonargon (Ar/Ar) sont adaptées aux minéraux riches en potassium, notamment dans les roches volcaniques, et permettent de dater des événements avec des précisions de $0,5 \%$ à $1 \%$. Les techniques reposant sur les séries de l'uranium, et ses descendants, impliquent un grand nombre de radionucléides de périodes différentes. Ces méthodes sont typiquement utilisées pour dater les matériaux du Pléistocène avec des précisions de l'ordre du pour cent. Elles sont appliquées aux coraux, qui enregistrent les varia-
Le carottier Calypso à bord du navire de recherche "Le Marion Dufresne". La mise au point de techniques de carottages sédimentaires toujours plus profonds permet de remonter l'échelle des temps. 


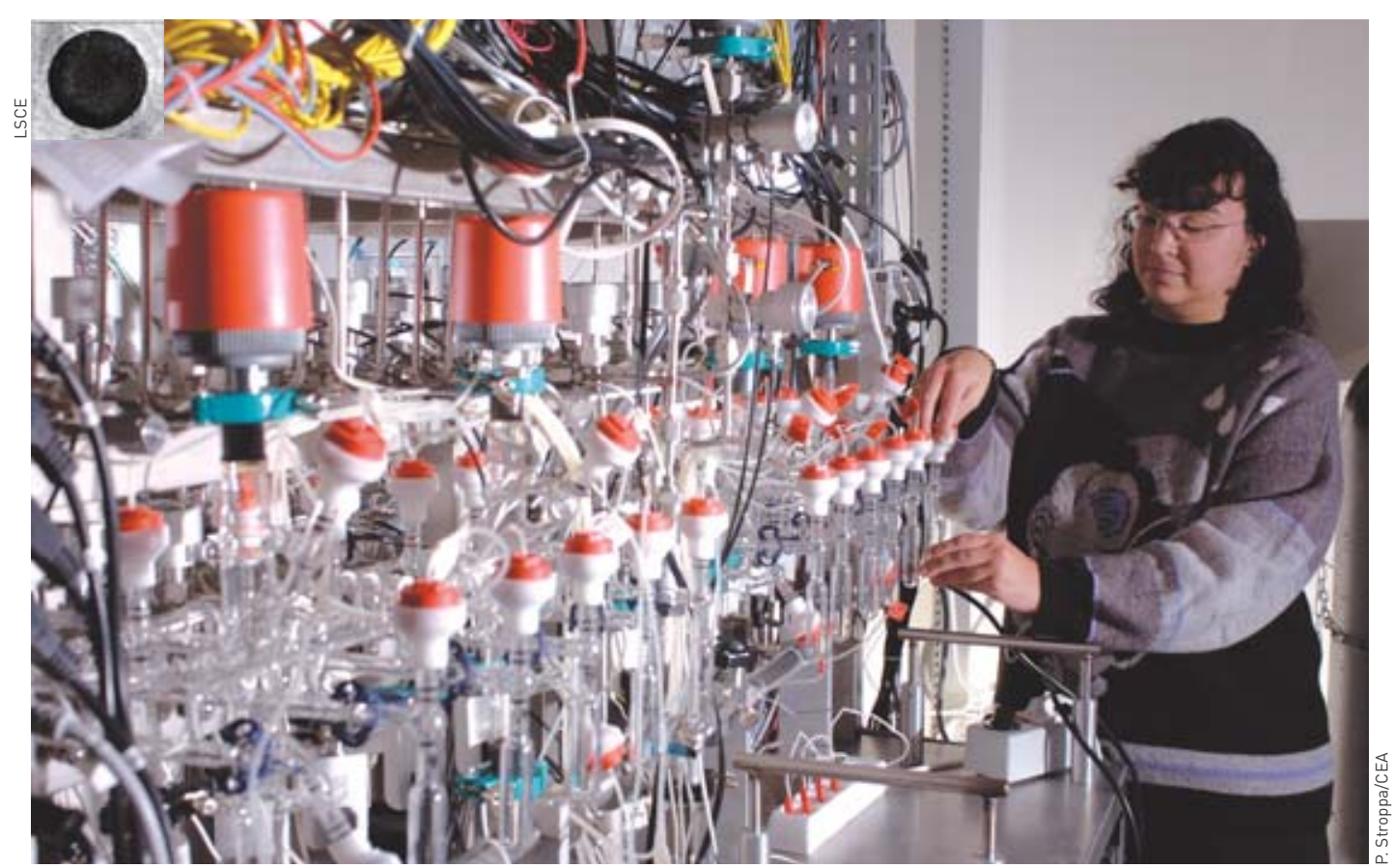

Le principe de la datation par le carbone 14 repose sur le fait que dans tout être vivant, le rapport de la concentration en ${ }^{14} \mathrm{C}$ à la concentration en ${ }^{12} \mathrm{C}$ est le même que dans le dioxyde de carbone atmosphérique. À sa mort, les échanges cessent. Le carbone 14 n'est plus renouvelé et sa radioactivité décroît avec une période de 5730 ans. En mesurant l'activité en ${ }^{14} \mathrm{C}$ de l'échantillon, il est possible d'en déduire son âge. La méthode par spectrométrie de masse par accélérateur présente l'avantage de n'utiliser que quelques centaines de microgrammes de carbone pour une analyse. Les échantillons, par exemple des foraminifères, sont traités chimiquement pour en extraire le carbone qui sera purifié sous forme de $\mathrm{CO}_{2}$. Le dioxyde de carbone sera ensuite réduit pour être transformé en graphite. Ici, mise en place des ampoules de $\mathrm{CO}_{2}$ sur le banc de réduction de l'installation Artémis. En haut à gauche, cible de graphite (de diamètre 1 mm environ).

tions du niveau marin et les changements physicochimiques des masses d'eau océanique, ainsi qu'aux stalagmites, qui archivent les conditions thermiques et pluviométriques. D'autres nucléides, comme le plomb $210\left({ }^{210} \mathrm{~Pb}\right)$ ou le radium $226\left({ }^{226} \mathrm{Ra}\right)$, rendent possible de dater des événements plus récents, en raison de leur période respective de 22 ans et 1600 ans. Ils sont adaptés aux minéraux inorganiques et biogènes ainsi qu'aux restes organiques. Ces techniques s'appuient sur la mesure des radionucléides initiaux et de leurs descendants, ce qui n'est pas envisageable pour la méthode par le carbone 14. En effet, ce nucléide se désintègre avec une période de 5730 ans en azote $14\left({ }^{14} \mathrm{~N}\right)$, et ce dernier ne peut être distingué de l'azote 14 de l'air $\left({ }^{14} \mathrm{~N} / \mathrm{N} \sim 0,99\right)$. De plus, la teneur en ${ }^{14} \mathrm{C}$ de l'atmosphère a varié au cours du temps, en raison des fluctuations de l'activité solaire, du champ magnétique terrestre, du temps de résidence et des échanges de carbone 14 entre les différents réservoirs de carbone. Les âges mesurés par

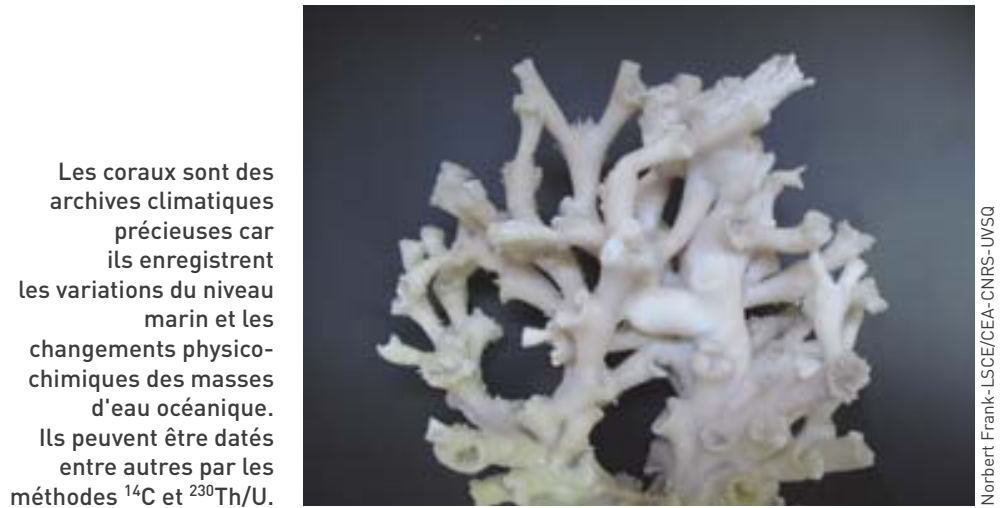

datation par le carbone 14 présentent donc un écart avec les âges absolus. Le croisement des mesures ${ }^{14} \mathrm{C}$ avec les âges dendrochronologiques ou avec ceux obtenus à partir d'autres nucléides radioactifs, principalement le couple ${ }^{230} \mathrm{Th} / \mathrm{U}$, donne accès à la quantification précise de cet écart, ou calibrage. Cette méthode, qui a valu le prix Nobel de Chimie à l'Américain Willard Frank Libby en 1960, permet de dater jusqu'à 50000 ans environ, avec des précisions de l'ordre de $0,5 \%$ à $2 \%$ en fonction de l'âge, des échantillons provenant des milieux océaniques, tels les foraminifères, coraux, coquillages, et des milieux continentaux, avec les cristaux inorganiques et biogènes (aragonite, calcite) et les restes organiques (graines, bois, charbons, insectes).

Aux mesures d'âge basées sur la radioactivité naturelle se sont ajoutés des marqueurs temporels radioactifs. En effet, certains nucléides ont été injectés en grandes quantités dans l'atmosphère lors des essais nucléaires au début des années 1960, comme le ${ }^{14} \mathrm{C}$, le césium $137\left({ }^{137} \mathrm{Cs}\right)$ - de période 30 ans -, ou le tritium - de période 12 ans -, ou bien encore lors de l'accident de Tchernobyl (Ukraine) en 1986 pour le ${ }^{137}$ Cs.

\section{Tirer profit de la désintégration} de radionucléides

Les méthodes radiogéniques s'appuient sur les défauts physiques laissés dans les minéraux, comme les traces de fission engendrées par les noyaux de recul (4)

\footnotetext{
(4) La scission du noyau d'uranium 238 en deux fragments (fission) libère une énergie, qui s'exprime principalement sous forme d'énergie cinétique. Les fragments sont éjectés à travers le matériau en créant des zones de dommage intense, appelées traces.
} 
lors de la désintégration de l'uranium 238. Cette technique renseigne essentiellement sur l'histoire thermique des roches, avec un domaine d'applicabilité sur les verres volcaniques qui constituent des marqueurs temporels régionaux uniques reliant les différentes archives sédimentaires. Elles concernent aussi les méthodes de luminescence stimulée optiquement (OSL) ou thermiquement (TL) qui consistent en une dosimétrie des radiations internes et externes subies naturellement par les cristaux en raison de la présence de nucléides radioactifs $\left({ }^{238} \mathrm{U},{ }^{232} \mathrm{Th}\right.$ et $\left.{ }^{40} \mathrm{~K}\right)$ contenus dans un cristal et son environnement. Ces techniques de luminescence s'appliquent aux cristaux de quartz et feldspaths inclus dans les roches volcaniques, les sédiments marins, les formations dunaires et les formations glaciaires loessiques (5). Elles sont théoriquement adaptées au Pléistocène avec des précisions de l'ordre de $5 \%$ en moyenne.

\section{Corréler les signaux similaires}

Les méthodes de chronologie relative sont basées sur la corrélation de séries sédimentaires qui présentent des caractéristiques physiques, chimiques ou biologiques communes identifiables dans des contextes régionaux ou globaux. Ainsi, les variations de la composition isotopique de l'oxygène des carbonates d'origine marine et continentale ou celles du $\mathrm{CO}_{2}$ piégé dans les bulles d'air des glaces montrent à l'échelle du globe des oscillations quasi-périodiques, attribuées aux changements d'insolation de la Terre au cours du temps. Celle-ci est en effet modifiée en fonction des paramètres orbitaux de la Terre ${ }^{(6)}$ dont l'orbite varie avec des cycles de 100000 ans (excentricité), ainsi que l'inclinaison de son axe de rotation avec l'obliquité (40 000 ans) et la précession (23000 ans et 19000 ans). Les variations d'insolation peuvent être calculées très précisément pour les temps passés, et les variations climatiques à long terme sont ainsi datées grâce à leur corrélation avec les cycles astronomiques de l'insolation. Cette méthode de corrélation ou orbital tuning a permis de dater les séries sédimentaires sur les derniers 25 millions d'années avec une précision de l'ordre de quelques pour cent.

De même, la polarité et l'intensité du champ magnétique terrestre ont varié au cours du temps. Leur

\footnotetext{
(5) Lœss: roche sédimentaire détritique meuble résultant, au cours du Pléistocène, de l'accumulation au sol, sous climat froid et sec, de limons transportés par le vent depuis des zones sources (alluvions, dépôts fluvio-glaciaires, sédiments côtiers et estuariens, zones arides) soumises à une érosion éolienne. Il est principalement formé de quartz détritique et de carbonate de calcium.

(6) Les paramètres orbitaux de la Terre définissent sa position dans l'espace par rapport au Soleil. La Terre est soumise à l'attraction du Soleil mais aussi des autres planètes du système solaire, ce qui a pour effet de perturber son mouvement. Trois types de modifications affectent la position de la Terre par rapport au Soleil: l'ellipse décrite par la Terre se déforme (excentricité); l'axe de rotation de la Terre observe une

inclinaison par rapport à la normale au plan de l'écliptique (le plan qui contient l'orbite terrestre ainsi que le Soleil), selon un angle qui varie entre $22^{\circ}$ et $25^{\circ}$ (obliquité); cet axe de rotation subit de ce fait un lent mouvement de déplacement (précession), décrivant un cône autour de la normale à l'écliptique, d'ouverture égale à l'obliquité (actuellement égale à $23^{\circ} 27^{\prime}$ ), et dont le sommet est au centre de la Terre. Ce qui a pour conséquence que les positions des équinoxes et des solstices, sur l'orbite terrestre, se déplacent graduellement, par rapport au périhélie (qui est le point d'insolation maximale).
}

étude à partir de roches volcaniques datées par les méthodes K/Ar et Ar/Ar a permis d'établir une chronologie de référence couvrant les derniers 100 millions d'années (Geomagnetic Polarity Time Scale, GPTS). Au cours du Pléistocène, se sont produites les inversions de polarité de Jaramillo ( 1,8 million d'années) et Brunhes-Matuyama (760 000 ans) ainsi que les excursions - instabilités de plus courte durée - tels, par exemple, les événements du Blake (115000 ans), Laschamp (41 000 ans) et Mono Lake ( 30000 ans). Les variations d'intensité du champ magnétique sont enregistrées en continu dans les sédiments marins, et la similitude de ces variations aux échelles globales ou régionales a permis d'établir des courbes chronostratigraphiques de référence s'étendant jusqu'à environ 800000 ans, grâce à l'identification et à la datation des inversions et excursions du champ magnétique. De même, l'archéomagnétisme, qui mesure le magnétisme rémanent d'objets historiquement connus, propose une méthode de datation d'objets couvrant les derniers 2000 ans. Les formations de sapropèles dans l'environnement méditerranéen, dépôts enrichis en matière organique, ainsi que les produits des éruptions volcaniques ou tephra constituent autant de marqueurs temporels visant à l'élaboration d'un cadre chronologique unifié.

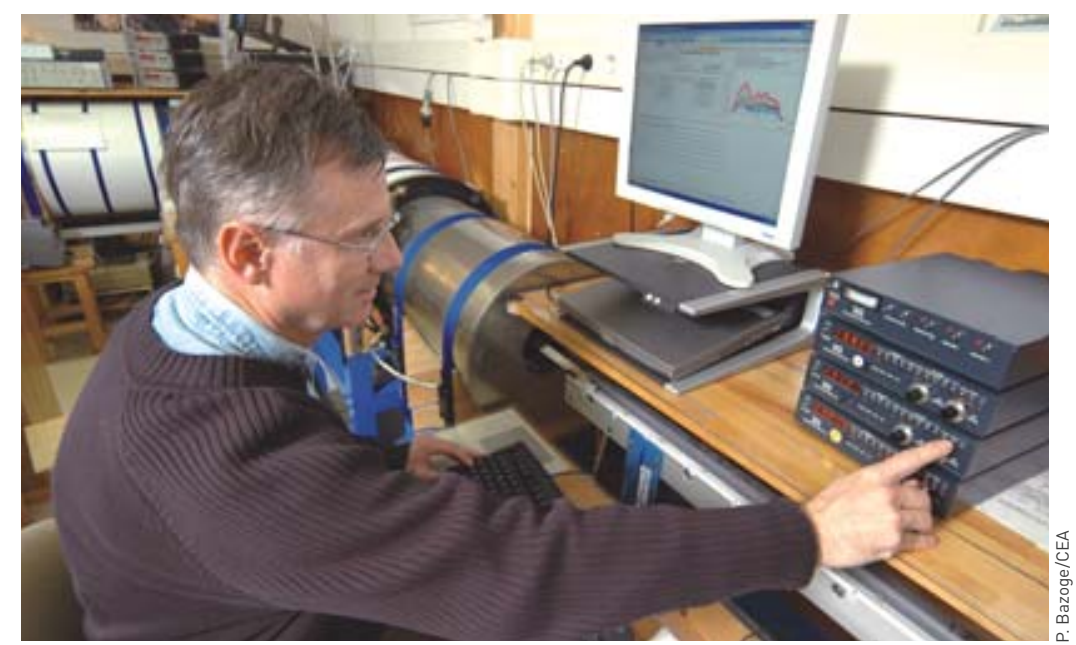

Mesure de l'aimantation rémanente d'un échantillon de sédiments marins.

Les sédiments acquièrent une aimantation rémanente lorsque, au moment de leur dépôt, des microparticules dotées d'un moment magnétique s'orientent statistiquement dans le champ magnétique ambiant. Les sédiments peuvent donc renseigner sur la direction et l'intensité du champ magnétique terrestre passé.

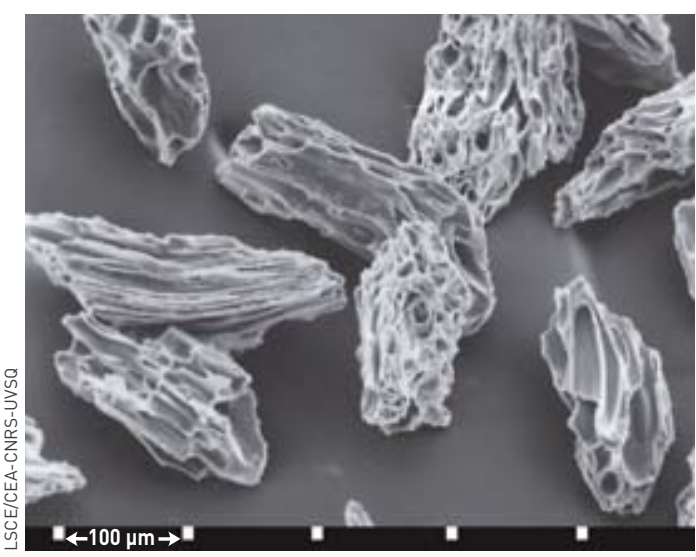

Image obtenue par microscopie électronique à balayage de verres volcaniques prélevés dans un tephra. Ces marqueurs temporels participent à l'élaboration d'un cadre chronologique global. 


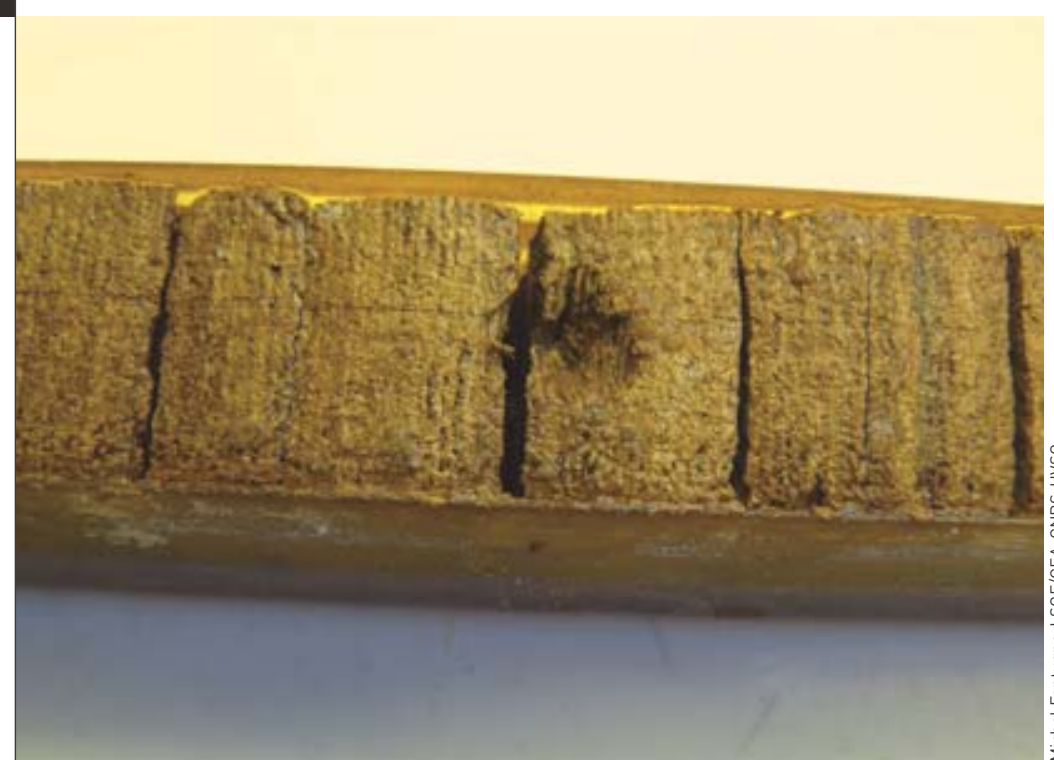

Les dépôts varvés sont préservés dans les sédiments en raison de la quasi-absence d'oxygène, et donc d'organismes fouisseurs au fond des lacs ou bassins océaniques dans lesquels ils sont observés. Chaque varve est constituée de deux lamines: une claire déposée l'été et une noirâtre l'hiver, le rythme de ces dépôts étant annuel. Les lamines de couleur claire se forment pendant l'été lorsque la sédimentation est la plus active du fait que l'alimentation par les eaux de fonte des glaciers est élevée. Le comptage de ces lamines dans les lacs proglaciaires (lacs au front d'un glacier en récession) permet de dater les sédiments jusqu'à 13000 ans environ. En associant ces comptages à la datation par le carbone 14 des pollens, graines ou restes d'insectes inclus dans ces sédiments, il devient ainsi possible d'estimer la différence entre les âges carbone 14 et les âges absolus. Ici, une carotte de sédiments varvés extraite du lac Perespilno en Pologne.

\section{Des mécanismes climatiques mieux compris}

La théorie astronomique des climats, émise pendant le XIX ${ }^{\mathrm{e}}$ siècle par le mathématicien français JosephAlphonse Adhémar et développée par la suite en particulier grâce aux calculs d'insolation effectués par le mathématicien et astronome serbe Milutin Milankovitch entre 1920 et 1940, a longtemps été rejetée par la communauté scientifique. Les premières preuves en faveur de cette théorie proviennent de datations ${ }^{230} \mathrm{Th} / \mathrm{U}$ de coraux récifaux, prélevés dans les terrasses coralliaires de l'île Barbade (Petites Antilles). Dès 1968, le paléo-océanographe américain Wallace Smith Broecker et ses collègues avaient démontré que les âges de trois épisodes de haut niveau marin, résultant de la fonte des glaces à 80000 ans, 100000 ans et 120000 ans, correspondaient à ceux d'une insolation maximale dans l'hémisphère Nord. Cette théorie fut définitivement acceptée après que James D. Hays, John Imbrie et Nicholas John Shackleton eurent démontré, en 1976, l'existence de cycles astronomiques à partir des variations isotopiques de l'oxygène des foraminiferes prélevés dans

(7) K. A. Hughen, M. G. L. Baillie, E. Bard,

J. W. Beck, C. J. H. Bertrand, P. G. Blackwell, C. E. Buck, G. S. Burr, K. B. CutLer, P. E. DAMON, R. L. EDWARDS, R. G. Fairbanks, M. Friedrich, T. P. Guilderson, B. Kromer, F. G. McCormac, S. W. Manning, C. Bronk Ramsey, P. J. Reimer, R. W. Reimer, S. Remmele, J. R. Southon, M. Stuiver, S. Talamo, F. W. TaYlor, J. van der Plicht and C. E. WeyhenmeYer, "Marine04 Marine radiocarbon age calibration, 0 - $26 \mathrm{cal} \mathrm{kyr}$ BP”, Radiocarbon, 46, p. 1059-1086, 2004.

(8) T. Blunier, J. Chappellaz, J. Schwander, A. Dällenbach, B. Stauffer, T. F. Stocker, D. Raynaud, J. Jouzed,

H. B. Clausen, C. U. Hammer and S. J. Johnsen, "Asynchrony of Antarctic and Greenland climate change during the last glacial period", Nature, 394, p. 739-743, 1998 des carottages du Pacifique Sud avec les périodicités de 106000 ans, 43000 ans, 24000 ans et 19000 ans. La chronologie de ces variations reposait sur des âges obtenus par les méthodes carbone 14, Th/U et K/Ar, l'âge K/Ar datant le renversement de polarité du champ magnétique Brunhes-Matuyama à environ 700000 ans.

La géochronologie a aussi contribué à modifier notre compréhension du fonctionnement de la boucle de circulation océanique globale, qui décrit la formation des eaux profondes dans l'océan Nord Atlantique, puis leur trajet vers l'océan Pacifique et enfin leur retour en surface vers l'océan Atlantique. Des fluctuations rapides du climat ponctuent la dernière période glaciaire et la déglaciation, avec les événements froids du Dryas récent et de Heinrich ou bien les alternances climatiques chaudes et froides des événements de Dansgaard/Oeschger. Elles étaient expliquées par un mode "on" ou "off" de la formation des eaux profondes dans l'océan Nord Atlantique, le mode "off" ayant pour effet de limiter les transferts de chaleur dans l'océan et les échanges entre l'océan et l'atmosphère aux hautes latitudes. Ce scénario a été infirmé, notamment grâce aux mesures précises de la teneur en ${ }^{14} \mathrm{C}$ de l'atmosphère, obtenue au cours de la déglaciation par le croisement des âges ${ }^{14} \mathrm{C}$ et des âges absolus déduits du comptage des varves sédimentaires déposées annuellement dans le bassin de Cariaco, Venezuela (7). Le $\mathrm{CO}_{2}$ de l'atmosphère est transféré vers les couches profondes de l'océan via la circulation océanique et, en raison de la très faible abondance $\mathrm{du}{ }^{14} \mathrm{C}\left({ }^{14} \mathrm{C} / \mathrm{C} \sim 10^{-14}\right)$, la teneur en ${ }^{14} \mathrm{CO}_{2}$ de l'atmosphère est très sensible au taux de formation des eaux profondes. Au cours de l'événement climatique froid du Dryas récent, dont la durée est d'environ 1200 ans calendaires, la teneur en ${ }^{14} \mathrm{C}$ de l'atmosphère a d'abord augmenté pendant les premiers 200 ans en accord avec un schéma de réduction de la formation des eaux profondes dans l'océan Nord Atlantique. Par contre, ce schéma ne pouvait expliquer la diminution $d u{ }^{14} \mathrm{C}$ notée au cours des 1000 ans suivants dans un contexte de climat froid. La synchronisation des compositions isotopiques de l'oxygène par la mesure du méthane dans les glaces du Groenland et de l'Antarctique ${ }^{(8)}$ montrait que les changements climatiques abrupts observés à la fois au Nord et au Sud étaient en opposition de phase. Lorsque l'Antarctique était plus chaud, le Groenland était froid, et inversement. Analysant ces deux résultats, Wallace Smith Broecker proposa en 1998 un schéma de bascule du transfert Nord-Sud de chaleur ou thermal bipolar see-saw: les périodes chaudes en Antarctique étaient l'expression de la formation d'eaux profondes dans l'océan Austral, permettant ainsi une diminution de la teneur en ${ }^{14} \mathrm{C}$ de l'atmosphère. Dans l'hémisphère Nord, un climat froid, modulé par l'arrêt de la formation des eaux profondes dans l'océan Nord Atlantique prédominait pendant le même intervalle de temps.

$>$ Martine Paterne

Laboratoire des sciences du climat et de l'environnement/Institut Pierre-Simon Laplace Unité mixte de recherche CEA-CNRS-UVSQ Direction des sciences de la matière CEA Centre de Saclay (Gif-sur-Yvette) 